\title{
The association between total duration of breastfeeding and serum 25-hydroxyvitamin D
}

Denise Darmawikarta, MPH, ${ }^{a}$ Yang Chen, MA, MSc, ${ }^{\mathrm{a}}$ Gerald Lebovic, PhD, ${ }^{\mathrm{a}}$ Catherine S.

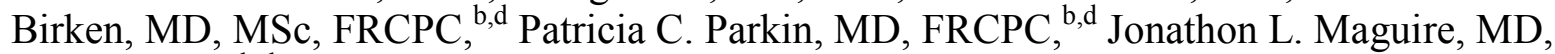
MSc, FRCPC ${ }^{\text {b,d,e }}$ on behalf of the TARGet Kids! Collaboration*

Affilitations: ${ }^{\mathrm{a}}$ The Applied Health Research Centre of the Li Ka Shing Knowledge Institute at St. Michael's Hospital, University of Toronto; ${ }^{b}$ Pediatrics Outcomes Research Team, Division of Paediatric Medicine, Department of Paediatrics, The Hospital for Sick Children; ${ }^{\circ}$ Department of Preventive Dental Science, Faculty of Dentistry, University of Manitoba; ${ }^{\mathrm{d}}$ Department of Pediatrics, Faculty of Medicine, University of Toronto; 'Department of Pediatrics, St. Michael's Hospital, Toronto, Ontario, Canada

Address correspondence to: Jonathon Maguire, MD, MSc, FRCPC, Department of Pediatrics, St Michael's Hospital, 30 Bond St, 15-CC-014, Toronto ON M5B 1W8 Canada. Email: jonathon.maguire@utoronto.ca

Short title: Total duration of breastfeeding and serum 25 -hydroxyvitamin D

Abbreviations: 25-OHD - 25-hydroxyvitamin D; AAP - American Academy of Pediatrics; CPS - Canadian Paediatric Society; SFP - Société Française de Pédiatrie (French Society of Pediatrics); CI - 95\% confidence interval; OR - odds ratio; BMI - body mass index; NHANES National Health and Nutrition Examination Survey

Keywords: breastfeeding, vitamin D, vitamin D supplementation, children

Funding source: Supported by the Canadian Institutes of Health Research MOP grant 106532 and St Michael's Foundation. The Pediatrics Outcomes Research Team is supported by the Hospital for Sick Children Foundation. These funding organizations were not involved in design and conduct of the study; collection, management, analysis, and interpretation of the data; and preparation, review, or approval of the manuscript.

Financial disclosure: All authors have indicated that they have no financial relationships relevant to this article to disclose.

Conflict of Interest: All authors have no conflicts of interest to disclose. 
What's known on this subject: Exclusive breastfeeding is a risk factor for vitamin D deficiency. Little is known about the effect of total duration of breastfeeding, including the periods of exclusive breastfeeding as well as after the addition of complementary foods, on vitamin D status.

What this study adds: Our results suggest that breastfed children who supplemented with vitamin $\mathrm{D}$ beyond the first year are at increased risks of inadequate vitamin D status. This highlights the importance of promoting continued vitamin D supplementation during breastfeeding of any duration.

\section{Contributors' Statement:}

Ms Darmawikarta conceptualized and designed the study, designed the data collection instruments, analyzed and interpreted the data, performed statistical analysis, drafted the manuscript, and approved the final manuscript as submitted.

Mr Yang designed the data collection instruments, conceptualized and designed the study, designed the data collection instruments, analyzed and interpreted the data, performed statistical analysis, and critically reviewed the final manuscript.

Dr. Lebovic performed statistical analysis and critically revised and reviewed the manuscript for important intellectual content, and approved the final manuscript as submitted.

Dr Birken conceptualized and designed the study, designed the data collection instruments, analyzed and interpreted the data, critically revised and reviewed the manuscript for important intellectual content, and approved the final manuscript as submitted.

Dr Parkin conceptualized and designed the study, designed the data collection instruments, analyzed and interpreted the data, critically revised and reviewed the manuscript for important intellectual content, and approved the final manuscript as submitted.

Dr Maguire conceptualized and designed the study, designed the data collection instruments, analyzed and interpreted the data, drafted the manuscript, critically revised and reviewed the manuscript for important intellectual content, and approved the final manuscript as submitted.

All authors had full access to all of the data in the study and take responsibility for the integrity of the data and the accuracy of the data analysis. 


\section{ABSTRACT}

Objectives: To determine whether total duration of breastfeeding is associated with serum 25hydroxyvitamin D (25-OHD) level in early childhood and to explore the effect of vitamin D supplementation on the relationship between total duration of breastfeeding and vitamin D status.

Methods: A cross-sectional study of healthy children seen for primary health care between September 2011 and August 2013 was conducted through the TARGet Kids! practice-based research network. Adjusted linear regression was used to determine the association between total duration of breastfeeding and serum 25-OHD and to explore the effect of vitamin D supplementation. Adjusted logistic regression was used to assess the probability of 25-OHD $<20$ $\mathrm{ng} / \mathrm{mL}$ in supplemented vs. non-supplemented children.

Results: An association that was modified by vitamin D supplementation was identified between total duration of breastfeeding and serum 25-OHD ( $\mathrm{p}=0.0251)$. Every 1 month increase in total duration of breastfeeding was associated with a $0.11 \mathrm{ng} / \mathrm{mL}$ lower median serum 25-OHD level (95\% CI: $-0.20,-0.02 \mathrm{ng} / \mathrm{mL}$ ) among children who were not supplemented. The odds of serum $25-\mathrm{OHD}<20 \mathrm{ng} / \mathrm{mL}$ increased by $6 \%$ with every 1 month increase in total duration of breastfeeding among children were not supplemented $(\mathrm{OR}=1.06,95 \% \mathrm{CI}: 1.03,1.10)$. There was no statistically significant association between total duration of breastfeeding and 25-OHD among children who did receive vitamin D supplementation $(\mathrm{p}=0.43)$.

Conclusions: Breastfed children who do not receive vitamin D supplementation beyond the first year of life may be at an increased risk of inadequate vitamin D status. Vitamin D supplementation appears to mitigate this risk. These findings support the use of vitamin D supplementation during breastfeeding of any type and duration. 


\section{Introduction}

Vitamin D is a fat-soluble steroid that has numerous biological actions which affect health. ${ }^{1-4}$ In infants and young children, severe vitamin D deficiency results in rickets. ${ }^{5-8}$

Breastfed infants are at increased risks for developing vitamin D deficiency and rickets due to minimal vitamin D in breast milk. ${ }^{9}$ While previous research supports the association between exclusive breastfeeding and vitamin D deficiency, ${ }^{10,11}$ little is known about the effect of total duration of breastfeeding, which includes both the periods of exclusive breastfeeding and after the introduction of complementary foods, on vitamin D status.

Guidelines for vitamin D supplementation during breastfeeding vary in different parts of the world. The American Academy of Pediatrics (AAP) recommends that, "Any breastfeeding infant, regardless of whether he or she is being supplemented with formula, should be supplemented with 400 IU of vitamin D". ${ }^{12}$ The Committee on Nutrition of the French Society of Paediatrics (SFP) also suggests continuous vitamin D supplementation beyond the first year of life, ${ }^{13}$ irrespective of breastfeeding status, while the Canadian Paediatric Society (CPS) recommends $400 \mathrm{IU} /$ day of vitamin D supplementation for breastfed infants up to one year of age. ${ }^{14}$ Given the important role that both breastfeeding and vitamin D play on influencing the long-term health of infants and young children, such guideline variation suggests a need to better understand the relationship between total duration of breastfeeding and vitamin $\mathrm{D}$ status in the first years of life.

The primary objective of this study was to determine whether total duration of breastfeeding is associated with infant serum 25-hydroxyvitamin D (25-OHD) level in a cohort of healthy urban North American children. Our secondary objective was to explore the effect of vitamin D supplementation on the relationship between total duration of breastfeeding and vitamin D status.

\section{Methods}

\section{$\underline{\text { Study Design }}$}

This was a cross-sectional study involving healthy urban children age 0-5 years receiving primary health care at a TARGet Kids! participating family medicine or pediatrician's office between September 2011 and August 2013.

\section{Definitions}

Infant feeding practices have been classified through a variety of terms. For the purposes of this study, "exclusive breastfeeding" refers to feeding with only breast milk without any additional foods or liquids ${ }^{15}$ and "nonexclusive breastfeeding" refers to feeding with breast milk in addition to other fluids or complementary foods. The term "total duration of breastfeeding" refers to the duration of breastfeeding of any kind (including exclusive or nonexclusive).

\section{Study population}


Children were recruited by trained research personnel embedded in 7 TARGet Kids! participating pediatric and family medicine practices. TARGet Kids! is a collaboration between University of Toronto child health outcomes researchers and primary care physicians from the Department of Paediatrics and the Department of Family and Community Medicine to collect longitudinal data on common health problems affecting urban children. Sociodemographic, lifestyle and nutritional information were collected during a scheduled primary healthcare physician visit through a standardized parent-completed survey instrument based on the Canadian Community Health Survey. ${ }^{16}$ For children who had had more than one clinic visit, the first visit with serum vitamin D testing was included in the analysis.

Children with health conditions affecting growth (e.g. cystic fibrosis), those with chronic condition(s) (except for asthma) and those with severe developmental delay were excluded from the study.

\section{Outcome and predictor variables}

The primary outcome variable was serum 25 -hydroxyvitamin $\mathrm{D}$ level. This was measured from serum samples using a competitive 2-step chemiluminescence assay (DiaSorin LIAISON 25hydroxyvitamin D TOTAL) ${ }^{17}$ at in the Mount Sinai Services Laboratory in Toronto (mountsinaiservices.com). Extensive testing and validation of this machine has been performed and has demonstrated an intra-assay imprecision of $7.2 \%$ at a concentration of $85 \mathrm{ng} / \mathrm{mL}$ (to convert to nmol per liter, multiply by 2.496) and an interassay imprecision of $4.9 \%$ at $13 \mathrm{ng} / \mathrm{mL}$, $8.9 \%$ at $31 \mathrm{ng} / \mathrm{mL}$, and $17.4 \%$ at $85 \mathrm{ng} / \mathrm{mL}$, values that are well within acceptable limits for biochemical measurements. ${ }^{18,19}$

The primary predictor variable was total duration of breastfeeding, which was determined from the response to the question, "For how long has your child been breastfed?" Maternal recall has been found to be a valid and reliable estimate of breastfeeding duration. ${ }^{20}$ Children who had never breastfed were classified as having a total duration of breastfeeding of 0 months. Those currently breastfeeding were classified as having a total duration of breastfeeding equal to the child's current age.

Covariates were chosen for inclusion if they were associated with 25-hydroxyvitamin D in 1 or more published studies and included age, sex, body mass index (BMI) $z$-score, use of vitamin D supplementation, skin pigmentation, median after-tax household income, season of blood sampling (May-September vs. October-April), daily outdoor play time, and total daily cups of cow's milk and formula. Weight was measured using a precision digital scale $( \pm 0.025 \%$; SECA) and standing height was measured using a stadiometer (SECA). BMI $z$ scores were calculated using World Health Organization growth standards. ${ }^{21}$ The use of vitamin D supplementation was determined from the response to the questions "Does your child take a vitamin D-containing supplement regularly?" In Canada, commercially available vitamin D supplements contain 400IU vitamin D. Skin pigmentation was measured by a trained research assistant using the Fitzpatrick scale, a widely-used 6-category skin pigmentation classification system. ${ }^{22,23}$ Six-digit postal codes were used to obtain the median after-tax neighborhood household income for each participant (using the Statistics Canada Postal Code Conversion File and data from the 2006 Census). Ecological measures of socioeconomic status have been used as proxies for individuallevel measures of socioeconomic status. ${ }^{24-26}$ 


\section{$\underline{\text { Statistical Analysis }}$}

Descriptive statistics were generated for the main outcome and covariates. For our primary analysis, a multivariable linear regression model was developed to examine the relationship between total duration of breastfeeding and serum 25-OHD level. All predictor variables were included in the final model regardless of their associated $\mathrm{P}$ values in order to avoid potential bias of the R-squared and standard errors. ${ }^{27}$ Missing data were handled by multiple imputation using additive regression, bootstrapping and predictive mean matching. ${ }^{27}$ The maximum rate of missing data for any variable was $13 \%$. Log transformation of serum 25-OHD level was performed to meet the normality assumption.

Our secondary analysis was aimed at determining the effect of vitamin D supplementation on the relationship between total duration of breastfeeding and serum 25-OHD level. To accomplish this, we repeated the primary analysis with the inclusion of an interaction term between total duration of breastfeeding and vitamin D supplement use. Further, we developed a multivariable logistic regression model to explore the association between increasing duration of breastfeeding and the Institute of Medicine suggested serum 25-OHD level of $20 \mathrm{ng} / \mathrm{mL}$ among supplemented and non-supplemented children. ${ }^{28}$

All statistical tests were two-tailed and significance was considered at the 0.05 level. Statistical analyses were performed using SAS 9.3 (SAS Institute Inc, USA) for Windows (Microsoft Corporation, USA) and the R project for statistical computing.

Research ethics approval was granted through the Research Ethics Board of the Hospital for Sick Children and of St. Michael's Hospital. All parents of participating children consented to participation in the study.

\section{Results}

\section{Population}

Of the 4533 children aged 0-5 years old who participated in TARGet Kids! between September 2011 and August 2013, 2510 (55\%) of them had serum 25-OHD level obtained. Two of the children had abnormally high serum $25-\mathrm{OHD}$ levels $>140 \mathrm{ng} / \mathrm{mL}$ and were excluded from the final analysis as outliers (Fig 1). The median age of included children was 24.5 months (IQR 15.1-40.8 months) and 1330 (53\%) children were male. The median serum 25-OHD level was 32 $\mathrm{ng} / \mathrm{mL}$ (IQR $26-40 \mathrm{ng} / \mathrm{ml}$ ) and $6 \%$ of children had $25-\mathrm{OHD}<20 \mathrm{ng} / \mathrm{mL}$. The median total duration of breastfeeding was 10 months (IQR 6.13 months). 54\% of children who were breastfed for any duration received vitamin D supplementation. Population characteristics are presented in Table 1. Imputation for missing values did not change descriptive characteristics.

\section{Breastfeeding and serum 25-hydroxyvitamin D}

Univariate analysis revealed that total duration of breastfeeding was not significantly associated with serum 25-OHD. This association remained non-significant after adjusting for clinically 
relevant covariates (Table 2). However, the interaction between vitamin D supplementation and total duration of breastfeeding was statistically significant $(p=0.0251)$, suggesting vitamin $D$ supplementation as an effect modifier of the association between total duration of breastfeeding and serum 25-OHD (Table 2). This is shown graphically in Figure 2. Among children who did not receive vitamin D supplementation, every 1 month increase in total duration of breastfeeding was associated with a $0.11 \mathrm{ng} / \mathrm{mL}$ lower median serum 25-OHD level (95\% CI: $-0.2,-0.02$ $\mathrm{ng} / \mathrm{mL}, \mathrm{p}=0.0236)$. Among children who did receive vitamin $\mathrm{D}$ supplementation, total duration of breastfeeding was not significantly associated with 25-OHD ( $\mathrm{p}=0.43)$. Age, total daily milk consumption, skin type and season of blood sampling were statistically significant covariates (Table 2).

We also found a statistically significant association between total duration of breastfeeding and the odds of $25-\mathrm{OHD}<20 \mathrm{ng} / \mathrm{mL}$ among children who were not supplemented. For every 1 month increase in total duration of breastfeeding, the odds of $25-\mathrm{OHD}<20 \mathrm{ng} / \mathrm{mL}$ increased by $6 \%$ (adjusted $\mathrm{OR}=1.06,95 \% \mathrm{CI}$ : $1.03,1.10$ ) among those who did not receive supplementation.

The odds of $25-\mathrm{OHD}<20 \mathrm{ng} / \mathrm{mL}$ was not significantly associated with total duration of breastfeeding among children who did receive vitamin $\mathrm{D}$ supplementation $(\mathrm{OR}=0.97,95 \% \mathrm{CI}$ : $0.93,1.01)$.

The cumulative adjusted predicted probability of vitamin $\mathrm{D}<20 \mathrm{ng} / \mathrm{mL}$ is shown in Figure 3 for both supplemented and non-supplemented children. The predicted probability of vitamin $\mathrm{D}<20$ $\mathrm{ng} / \mathrm{mL}$ among children who did not receive supplementation was $16 \%$ with a total breastfeeding duration of 24 months and $28 \%$ with a total breastfeeding duration of 36 months.

\section{Discussion}

In this study, we have identified an association that was modified by vitamin D supplementation between total duration of breastfeeding and 25-OHD. Among children who did not receive vitamin D supplementation each additional month of total breastfeeding duration was associated with a $0.1 \mathrm{ng} / \mathrm{mL}$ lower 25-OHD. Further, the odds of $25-\mathrm{OHD}<20 \mathrm{ng} / \mathrm{mL}$ was $6 \%$ higher for every 1 month increase in total duration of breastfeeding such that by 2 years of age, the predicted probability of $25-\mathrm{OHD}<20 \mathrm{ng} / \mathrm{mL}$ was $16 \%$ and $28 \%$ at 3 years of age, among children who did not receive vitamin D supplementation. Among children who did receive vitamin D supplementation, we did not identify a relationship between total duration of breastfeeding and serum 25-OHD level. This suggests that the decline in serum 25-OHD during breastfeeding of any duration may be mitigated by supplementation with vitamin $\mathrm{D}$.

According to the World Health Organization (WHO), breastfeeding is recommended to continue to two years of age and beyond, as mutually desired by mother and child. ${ }^{29}$ While breast milk contains many of the required nutrients for supporting growth, it is widely accepted that breast milk does not provide adequate amounts of vitamin D. ${ }^{30-32}$ Exclusive breastfeeding in the first year without vitamin D supplementation is a known risk factor for severe vitamin D deficiency. ${ }^{10}$ Less is known about the relationship between total duration of breast feeding particularly beyond the first year of age and vitamin D status. 
Nutritional recommendations in childhood include the introduction of complementary foods beginning at 6 months of age, when the nutrients in breast milk alone are thought to be inadequate to support normal growth. ${ }^{29}$ While vitamin D intakes may increase as complementary foods are introduced into the child's diet, it has been identified that the mean intake of vitamin D through complementary foods fails to meet vitamin D requirements for most children at 7-12 months of age. ${ }^{33}$ While the AAP and the Committee on Nutrition of the SFP recommend vitamin D supplementation while breastfeeding within the first few days of life and continuing throughout childhood, ${ }^{12,13}$ the CPS recommends vitamin D supplementation while breastfeeding only up to 1 year of age. ${ }^{14}$ The latter has been attributed to a lack of evidence for $400 \mathrm{IU} /$ day of vitamin D in optimizing vitamin status in children. ${ }^{34}$ Our findings suggest that among children who did not receive vitamin D supplementation, longer total duration of breastfeeding was associated with lower 25-OHD and higher odds of $25-\mathrm{OHD}<20 \mathrm{ng} / \mathrm{mL}$, the Institute of Medicine recommended level. ${ }^{28}$ These observations support the AAP and SFP recommendations for continued vitamin D supplementation beyond the first year of life while breastfeeding.

Low uptake of vitamin D supplementation during breastfeeding has been previously attributed to lack of primary healthcare endorcement. ${ }^{35}$ Given that vitamin D supplementation rates have been shown to decline with age, ${ }^{36}$ primary healthcare endorsement of vitamin D supplementation may be particularly important among children with longer durations of breastfeeding.

To our knowledge, this is the first study to assess the effect of total duration of breastfeeding, not just the period of exclusive breastfeeding, on serum 25-OHD level. Strengths of our study include a relatively large sample size with comprehensive clinical data, which allowed for the adjustment of multiple social, demographic, biological and nutritional variables. Furthermore, our study was not limited to exclusive breastfeeding, but considered a broader and more inclusive definition of breastfeeding, which included the period after the introduction of complementary foods. This is important both clinically and for current health policy objectives supporting breastfeeding through the first 2 years of life and beyond. ${ }^{29}$ The relatively high prevalence of vitamin D supplementation in our study population is consistent with other jurisdictions. ${ }^{37}$ Additionally, our primary care venue allowed for the measurement of serum 25 OHD among children who routinely receive primary health care, which is a venue well suited for the promotion vitamin D supplementation during breastfeeding of any duration.

Limitations of our study include parent completed questionnaire data which may be subject to recall bias. We also did not account for unmeasured confounders, such as maternal vitamin D supplementation and dietary sources of vitamin D other than cow's milk and vitamin D supplementation, which may have introduced confounding. Further, while participation without blood testing was similar to other national surveys on breastfeeding, ${ }^{38,39}$ the lower participation with blood testing reflects the reality of conducting research requiring invasive procedures in young children.

\section{Conclusion}

In this study, we have identified an association between longer total duration of breastfeeding and lower serum 25-OHD that is modified by vitamin D supplementation. Serum 25-OHD appears to be maintained with vitamin D supplementation even with longer total duration of 
breastfeeding. Our findings support the AAP and SFP recommendations for vitamin D supplementation during breastfeeding of any duration. We suggest that primary health care is an ideal environment to support continued vitamin D supplementation in infants and children.

\section{Acknowledgements}

TARGet Kids! collaborators were as follows: Scientific Committee: Kawsari Abdullah, Laura Anderson, Yang Chen, Matthew D'Ascanio, Mikael Katz-Lavigne, Kanthi Kavikondala, Jieun (Grace) Lee, Jessica Omand, Navindra Persaud, Meta van den Heuvel, and Weeda Zabih; Site Investigators: Jillian Baker, Tony Barozzino, Joey Bonifacio, Douglas Campbell, Sohail Cheema, Brian Chisamore, Karoon Danayan, Paul Das, Mary Beth Derocher, Anh Do, Michael Dorey, Sloane Freeman, Keewai Fung, Charlie Guiang, Curtis Handford, Hailey Hatch, Sheila Jacobson, Tara Kiran, Holly Knowles, Bruce Kwok, Sheila Lakhoo,Margarita Lam-Antoniades,

Eddy Lau, Fok-Han Leung, Jennifer Loo, Sarah Mahmoud, Rosemary Moodie, Julia Morinis, Sharon Naymark, Patricia Neelands, James Owen, Michael Peer, Marty Perlmutar, Navindra Persaud, Andrew Pinto, Michelle Porepa, Nasreen Ramji, Noor Ramji, Alana Rosenthal, Janet Saunderson, Rahul Saxena,Michael Sgro, Susan Shepherd, Barbara Smiltnieks, Carolyn Taylor, Thea Weisdors, Sheila Wijayasinghe, Peter Wong, Ethel Ying, and Elizabeth Young.

We thank all participating families for their time and involvement in TARGet Kids! and are grateful to all practitioners who are currently involved in the TARGet Kids! research network. Steering Committee: Tony Barozzino, Brian Chisamore, Mark Feldman, andMoshe Ipp. Research Team: Charmaine Camacho, DiviyaElango, Julie DeGroot, Shanique Edwards, Nadia Kabir, Tarandeep Malhi, Juela Sejdo, Laurie Thompson, and Mandy Tran. Applied Health Research Centre: Gerald Lebovic, Magda Melo, and Patricia Nguyen. Mount Sinai Services Laboratory: Azar Azad. 


\section{References}

1. Hayes CE, Cantorna MT, DeLuca HF. Vitamin D and multiple sclerosis. Proc Soc Exp Biol Med. 1997;216(1):21-27.

2. Deeb KK, Trump DL, Johnson CS. Vitamin D signalling pathways in cancer: Potential for anticancer therapeutics. Nat Rev Cancer. 2007;7(9):684-700.

3. Hewison M. Vitamin D and the immune system: New perspectives on an old theme. Endocrinol Metab Clin North Am. 2010;39(2):365-79, table of contents.

4. Plum LA, DeLuca HF. Vitamin D, disease and therapeutic opportunities. Nat Rev Drug Discov. 2010;9(12):941-955.

5. Ozkan B. Nutritional rickets. J Clin Res Pediatr Endocrinol. 2010;2(4):137-143.

6. Unuvar T, Buyukgebiz A. Nutritional rickets and vitamin D deficiency in infants, children and adolescents. Pediatr Endocrinol Rev. 2010;7(3):283-291.

7. Pettifor JM. Nutritional rickets: Pathogenesis and prevention. Pediatr Endocrinol Rev. 2013;10 Suppl 2:347-353.

8. Prentice A. Nutritional rickets around the world. J Steroid Biochem Mol Biol. 2013;136:201206.

9. Ward LM, Gaboury I, Ladhani M, Zlotkin S. Vitamin D-deficiency rickets among children in canada. CMAJ. 2007;177(2):161-166.

10. Choi YJ, Kim MK, Jeong SJ. Vitamin D deficiency in infants aged 1 to 6 months. Korean J Pediatr. 2013;56(5):205-210.

11. Wall CR, Grant CC, Jones I. Vitamin D status of exclusively breastfed infants aged 2-3 months. Arch Dis Child. 2013;98(3):176-179.

12. Wagner CL, Greer FR, American Academy of Pediatrics Section on Breastfeeding, American Academy of Pediatrics Committee on Nutrition. Prevention of rickets and vitamin D deficiency in infants, children, and adolescents. Pediatrics. 2008;122(5):1142-1152.

13. Vidailhet M, Mallet E, Bocquet A, et al. Vitamin D: Still a topical matter in children and adolescents. A position paper by the committee on nutrition of the french society of paediatrics. Arch Pediatr. 2012;19(3):316-328.

14. Vitamin D supplementation: Recommendations for canadian mothers and infants. Paediatr Child Health. 2007;12(7):583-598. 
15. The World Health Organization. Exclusive breastfeeding. . http://www.who.int/elena/titles/exclusive breastfeeding/en/. Updated 2014. Accessed September 7, 2014.

16. Statistics Canada. Canadian Community Health Survey 2010. . www.statcan. gc.ca/access_acces/alternative_alternatif. action?1=eng\&loc=/imdb-bmdi/instrument/ 3226_Q1_V1-eng.pdf.

17. Diasorin, The Diagnostic Specialist website. . http://www.diasorin.com/en. Accessed July 29, 2014.

18. Maunsell Z, Wright DJ, Rainbow SJ. Routine isotope-dilution liquid chromatography-tandem mass spectrometry assay for simultaneous measurement of the 25-hydroxy metabolites of vitamins D2 and D3. Clin Chem. 2005;51(9):1683-1690.

19. Singh RJ, Taylor RL, Reddy GS, Grebe SK. C-3 epimers can account for a significant proportion of total circulating 25 -hydroxyvitamin $\mathrm{D}$ in infants, complicating accurate measurement and interpretation of vitamin D status. J Clin Endocrinol Metab. 2006;91(8):30553061.

20. Li R, Scanlon KS, Serdula MK. The validity and reliability of maternal recall of breastfeeding practice. Nutr Rev. 2005;63(4):103-110.

21. WHO child growth standards: methods and development. length/height-for-age, weight-forage, weight-for-length, weight-for-height and body mass index-for-age. World Health Organization Website Web site.

http://www.who.int/childgrowth/publications/technical_report_pub/en/. Updated 2006. Accessed July/12, 2014.

22. Fitzpatrick TB. The validity and practicality of sun-reactive skin types I through VI. Arch Dermatol. 1988;124(6):869-871.

23. Carpenter TO, Herreros F, Zhang JH, et al. Demographic, dietary, and biochemical determinants of vitamin D status in inner-city children. Am J Clin Nutr. 2012;95(1):137-146.

24. Krieger N. Overcoming the absence of socioeconomic data in medical records: Validation and application of a census-based methodology. Am J Public Health. 1992;82(5):703-710.

25. Mustard CA, Derksen S, Berthelot JM, Wolfson M. Assessing ecologic proxies for household income: A comparison of household and neighbourhood level income measures in the study of population health status. Health Place. 1999;5(2):157-171.

26. Soobader M, LeClere FB, Hadden W, Maury B. Using aggregate geographic data to proxy individual socioeconomic status: Does size matter? Am J Public Health. 2001;91(4):632-636. 
27. Harrell FE. Regression Modeling Strategies: With Applications to Linear Models, Logistic Regression, and Survival Analysi. 1st ed. New York: Springer; 2001.

28. Ross AC, Manson JE, Abrams SA, et al. The 2011 report on dietary reference intakes for calcium and vitamin D from the institute of medicine: What clinicians need to know. J Clin Endocrinol Metab. 2011;96(1):53-58.

29. World Health Organization. 55th World Health Assembly. Agenda Item 13.10: Infant and young child nutrition. . http://apps.who.int/gb/archive/pdf files/WHA55/ewha5525.pdf. Updated 2002. Accessed August 19, 2014.

30. Leerbeck E, Sondergaard H. The total content of vitamin D in human milk and cow's milk. Br J Nutr. 1980;44(1):7-12.

31. Hollis BW, Roos BA, Draper HH, Lambert PW. Vitamin D and its metabolites in human and bovine milk. J Nutr. 1981;111(7):1240-1248.

32. Reeve LE, Chesney RW, DeLuca HF. Vitamin D of human milk: Identification of biologically active forms. Am J Clin Nutr. 1982;36(1):122-126.

33. Friel JK, Hanning RM, Isaak CA, Prowse D, Miller AC. Canadian infants' nutrient intakes from complementary foods during the first year of life. BMC Pediatr. 2010;10:43-2431-10-43.

34. Roth DE. What should I say to parents about vitamin D supplementation from infancy to adolescence? Paediatr Child Health. 2009;14(9):575-577.

35. Taylor JA, Geyer LJ, Feldman KW. Use of supplemental vitamin d among infants breastfed for prolonged periods. Pediatrics. 2010;125(1):105-111.

36. Moore CE, Radcliffe JD, Liu Y. Vitamin D intakes of children differ by race/ethnicity, sex, age, and income in the united states, 2007 to 2010. Nutr Res. 2014;34(6):499-506.

37. Lepage MC, Moisan J, Gaudet M. What do quebec children eat during their first six months? Can J Diet Pract Res. 2004;65(3):106-113.

38. Zlotkin SH, Ste-Marie M, Kopelman H, Jones A, Adam J. The prevalence of iron depletion and iron-deficiency anaemia in a randomly selected group of infants from four canadian cities. Nutr Res. 1996;16(5):729-733.

39. Centers for Disease Control. Breastfeeding reportcard - United States, 2014. . http://www.cdc.gov/breastfeeding/data/reportcard.htm. Updated 2014. Accessed September 7, 2014. 


\section{Tables \& Figures}

Table 1. Baseline characteristics of study participants

\begin{tabular}{|c|c|c|}
\hline \multirow{2}{*}{ Characteristics } & \multicolumn{2}{|c|}{ Total Cohort $(\mathrm{N}=2508)$} \\
\hline & Frequency $(\%)$ & Median (IQR $\dagger)$ \\
\hline Total duration of breastfeeding (months) & & $10(6-13)$ \\
\hline 25-hydroxyvitamin D (ng/mL) & & $32(26-40)$ \\
\hline 25-hydroxyvitamin $\mathrm{D}<20 \mathrm{ng} / \mathrm{mL}$ & $157(6)$ & \\
\hline \multicolumn{3}{|l|}{ Supplementation } \\
\hline Yes & $1336(53)$ & \\
\hline No & $1140(45)$ & \\
\hline Age (months) & & $24.51(15.1-40.8)$ \\
\hline \multicolumn{3}{|l|}{ Sex } \\
\hline Male & $1329(53)$ & \\
\hline Female & $1179(47)$ & \\
\hline z-BMI & & $0.14(-3.99-7.93)$ \\
\hline \multicolumn{3}{|l|}{ Skin pigmentation } \\
\hline Type I & $314(13)$ & \\
\hline Type II & $956(38)$ & \\
\hline Type III & $709(28)$ & \\
\hline Type IV & $295(12)$ & \\
\hline Type V & $96(4)$ & \\
\hline Type VI & $58(2)$ & \\
\hline \multicolumn{3}{|l|}{ Season of blood sampling } \\
\hline May-September & $1219(49)$ & \\
\hline October-April & $1289(51)$ & \\
\hline \multicolumn{3}{|l|}{ Maternal ethnicity } \\
\hline European & $1726(69)$ & \\
\hline Asian (East, Southeast, South) & $399(16)$ & \\
\hline African/Caribbean/Latin American & $197(8)$ & \\
\hline Other & $61(2)$ & \\
\hline
\end{tabular}

Median household income (\$)

$55,105(42,935-68,951)$

Milk (cow's milk and formula) volume (cups)

$2(1-3)$ 
Total minutes of outdoor activities

$60(30-90)$

$\dagger$ IQR: Interquartile Range 
Table 2. Adjusted linear regression models for the association between total duration of breastfeeding and serum 25-hydroxyvitamin D

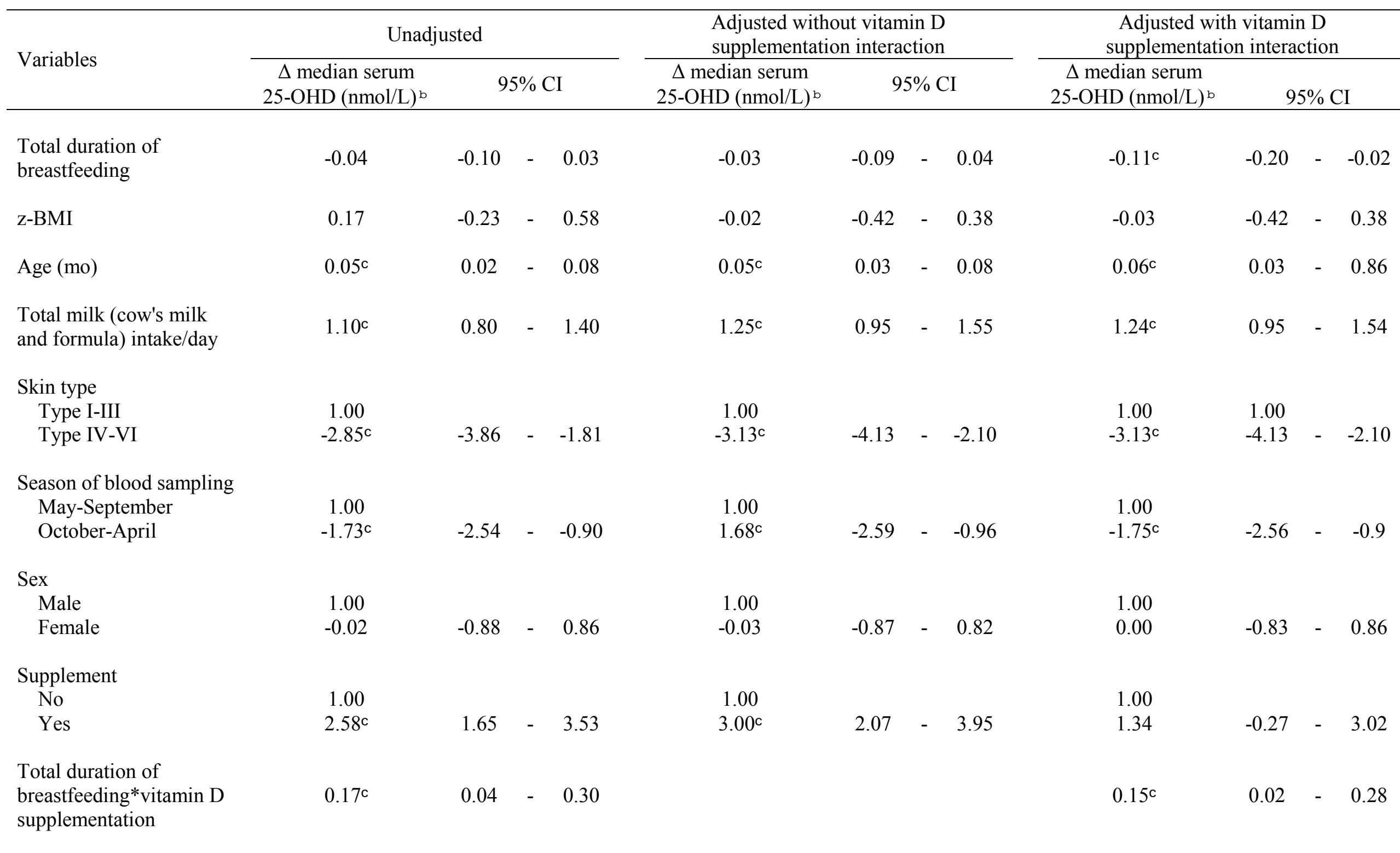

also adjusted for median after-tax household income and total minutes of outdoor activity per day

$\mathrm{b}$ negative values indicate a decrease in serum level, positive values indicate an increase in serum level

c $\mathrm{p}<0.05$ 
Figure 1. Participant flowchart.

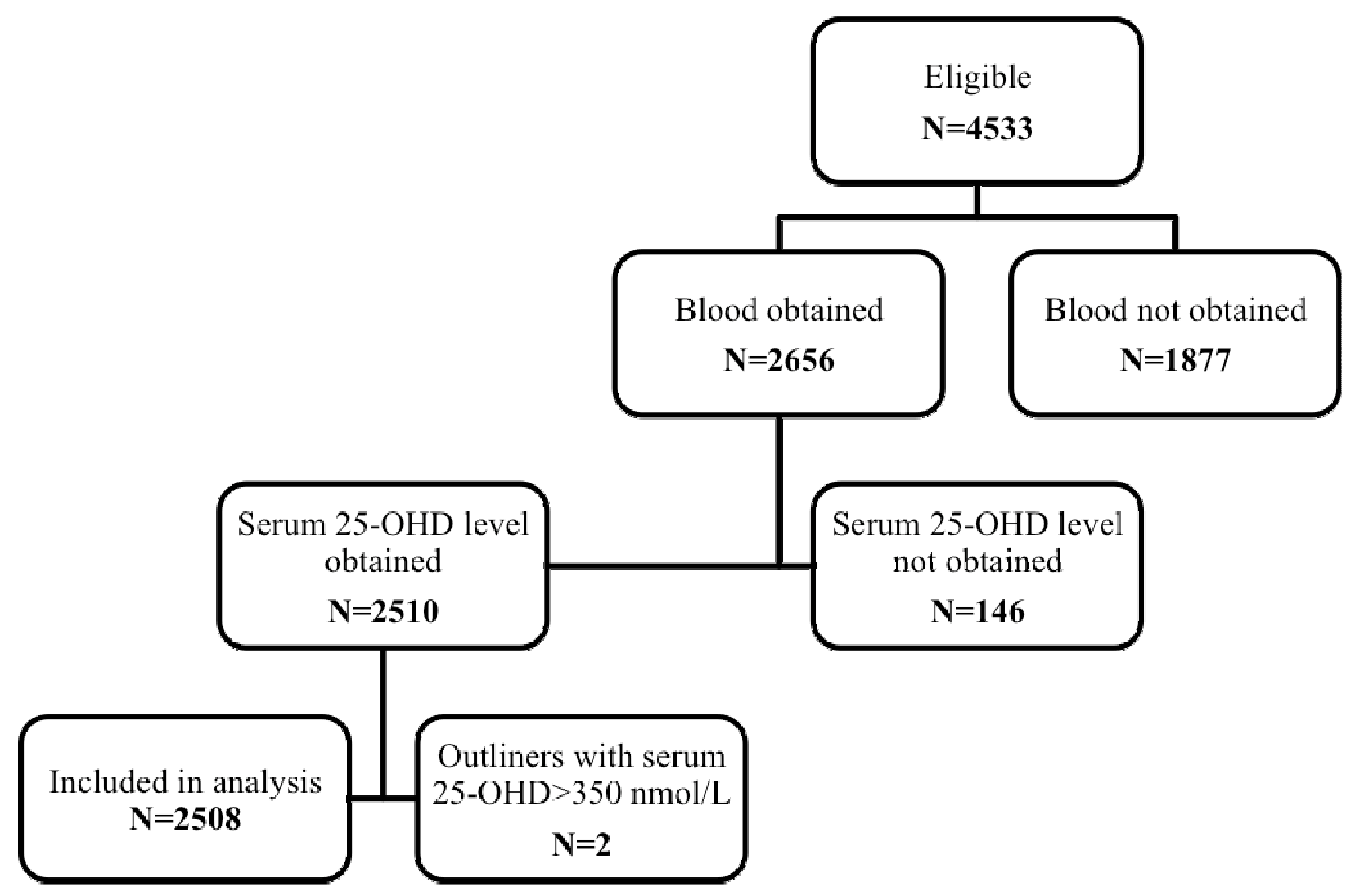


Figure 2. Serum 25-OHD as a function of total duration of breastfeeding.

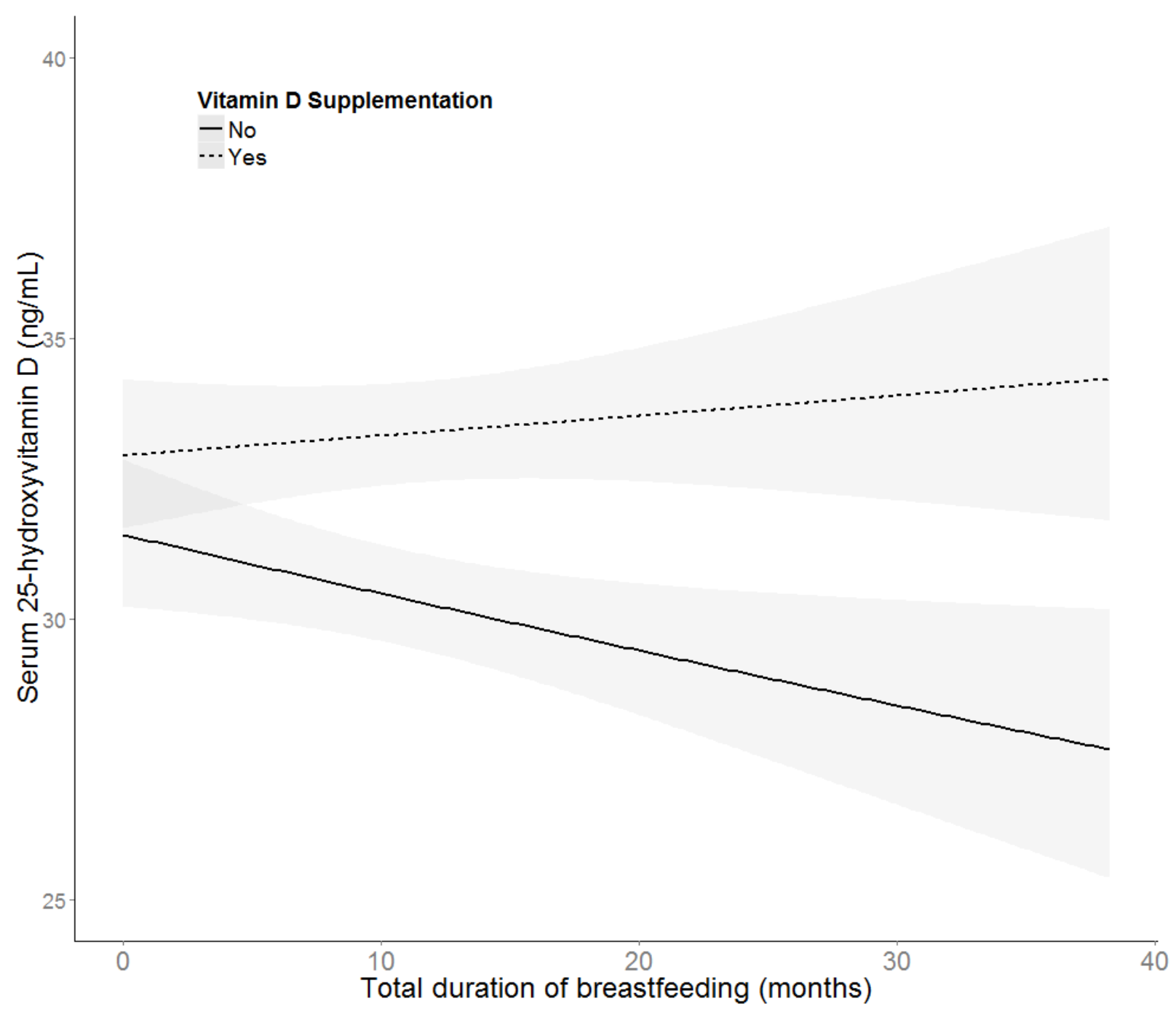

${ }^{¥}$ Adjusted for age, z-BMI, total milk, skin type, season of blood sampling, sex, median after-tax household income and total minutes of outdoor activities 
Figure 3. Cumulative predicted probability of vitamin $D<20 \mathrm{ng} / \mathrm{mL}$ as a function of total duration of breastfeeding.

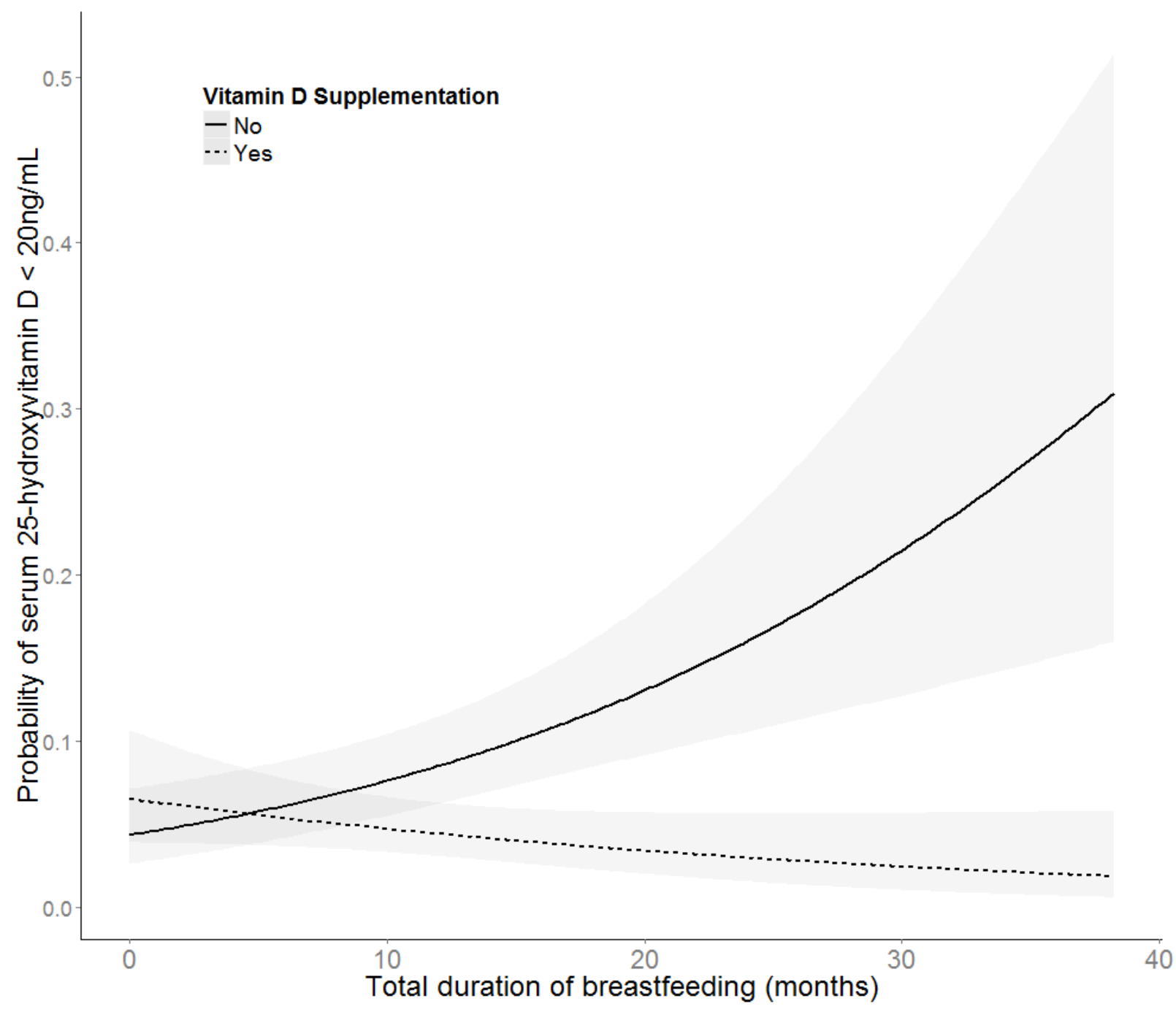

${ }^{¥}$ Adjusted for age, z-BMI, total milk, skin type, season of blood sampling, sex, median after-tax household income and total minutes of outdoor activities 